International Journal of Distributed and Parallel Systems (IJDPS) Vol.3, No.3, May 2012

\title{
Embedded system for Hazardous Gas detection and Alerting
}

\author{
V.Ramya ${ }^{1}$, B. Palaniappan ${ }^{2}$ \\ ${ }^{1}$ Assistant Professor, Department of CSE, Annamalai University, Chidambaram, India. \\ Email:ramyshrieyahoo.com \\ ${ }^{2}$ Dean, FEAT, Head, Department of CSE, Annamalai University, Chidambaram, India. \\ ${ }^{2}$ Email : bpau2002@yahoo.co.in
}

\begin{abstract}
Safety plays a major role in today's world and it is necessary that good safety systems are to be implemented in places of education and work. This work modifies the existing safety model installed in industries and this system also be used in homes and offices. The main objective of the work is designing microcontroller based toxic gas detecting and alerting system. The hazardous gases like LPG and propane were sensed and displayed each and every second in the LCD display. If these gases exceed the normal level then an alarm is generated immediately and also an alert message (SMS) is sent to the authorized person through the GSM. The advantage of this automated detection and alerting system over the manual method is that it offers quick response time and accurate detection of an emergency and in turn leading faster diffusion of the critical situation.
\end{abstract}

Keywords- Air pollution Monitoring, gas sensors, GSM module, wireless networks.

\section{INTRODUCTION}

The increase in the development of technology and the human race, we failed to take care about the surroundings in which we live in. Thus we polluted the environment and thereby reducing the quality of the place we live. Even though there are several aspects of pollution such as soil, air and water pollution, out of these air pollution acts as the serious aspect as the other can detected visually and by taste, but the polluted air cannot be detected as it can be odorless, tasteless and colorless. Hence there is a growing demand for the environmental pollution monitoring and control systems. In the view of the ever-increasing pollution sources with toxic chemicals, these systems should have the facilities to detect and quantify the sources rapidly. Toxic gases are one that causes serious health impacts, but are also used in industries in large quantities. These gases have to be monitored; such that increase in the normal level of them could be known and proper precaution measures can be taken. But the current systems available are not so portable and are costly and difficult to implement. So an embedded system is designed using PIC 16F877 Microcontroller, for the purpose of detection of hazardous gas leakage, which in turn avoids the endangering of human lives. The hazardous gases like LPG and propane were considered here. If these hazardous gases level exceeds normal level that is LPG $>1000$ ppm or Propane $>10000$ ppm then an alarm is generated immediately, and a SMS is sent to the authorized user as an alert message, which leads to faster diffusion of emergency situation. The system is affordable and can be easily implement in the chemical industries and in residential area which is surrounded by the chemical industries or plants, to avoid endangering of human lives. The system also supports to provide real-time monitoring of concentration of the gases which presents in the air. As this method is automatic the information can be given in time such that the endangering of human lives can be avoided. 
International Journal of Distributed and Parallel Systems (IJDPS) Vol.3, No.3, May 2012

\subsection{Related work}

In the year of 2008, LIU zhen-ya, WANG Zhen-dong and CHEN Rong, "Intelligent Residential Security Alarm and Remote Control System Based On Single Chip Computer", the paper focuses on, Intelligent residential burglar alarm, emergency alarm, fire alarm, toxic gas leakage remote automatic sound alarm and remote control system, which is based on 89c51 single chip computer. The system can perform an automatic alarm, which calls the police hotline number automatically. It can also be a voice alarm and shows alarm occurred address. This intelligent security system can be used control the electrical power remotely through telephone [8].

In the year of 2008, Chen Peijiang and Jiang Xuehhua, "Design and implementation of Remote Monitoring System Based on GSM", this paper focuses on the wireless monitoring system, because the wireless remote monitoring system has more and more application, a remote monitoring system based on SMS through GSM. Based on the overall architecture of the system, the hardware and software architecture of the system is designed. In this system, the remote signal is transmitted through GSM network. The system includes two parts which are the monitoring centre and the remote monitoring station. The monitoring centre consists of a computer and a TC35 communication module for GSM. The computer and the TC35 are connected by RS232. The remote monitoring station consist of a TC35 communication module for GSM, a MSP430F149 MCU, a display unit, sensors and a data gathering and processing unit. The software for the monitoring center and the remote monitoring station were designed using VB [7].

In the year of 2006, Ioan Lita, Ion Bogdan Cioc and Daniel Alexandru Visan, "A New Approach of Automatic Localization System Using GPS and GSM/GPRS Transmission", this paper focuses on, a low cost automotive localization system using GPS and GSM-SMS services, which provides the position of the vehicle on the driver's or owner's mobile phone as a short message (SMS) on his request. The system can be interconnected with the car alarm system which alerts the owner, on his mobile phone, about the events that occurs with his car when it is parked. The system is composed by a GPS receiver, a microcontroller and a GSM phone. In additional the system can be settled for acquiring and transmitting the information, whenever requested about automobile status and alerts the user about the vehicle started engine. The system can be used as a low cost solution for automobile position localizing as well as in car tracking system application [16].

In the year of 2002, K. Galatsis, W. Wlodarsla, K. Kalantar-Zadeh and A. Trinchi, "Investigation of gas sensors for vehicle cabin air quality monitoring", this paper focuses on, car cabin air quality monitoring can be effectively analyzed using metal oxide semiconducting (MOS) gas sensors. In this paper, commercially available gas sensors are compared with fabricated Moo3 based sensors possessed comparable gas sensing properties. The sensor has response $74 \%$ higher relative to the hest commercial sensor tested [21]. In the year 2000, K. Galatsis, W. Woldarsla, Y.X. Li and K. Kalantar-zadeh, "A Vehicle air quality monitor using gas sensors for improved safety", this paper focuses on A vehicle cabin air quality monitor using carbon monoxide ( $\mathrm{CO}$ ) and oxygen (02) gas sensors has been designed, developed and on-road tested. The continuous monitoring of oxygen and carbon monoxide provides added vehicle safety as alarms could be set off when dangerous gas concentrations are reached, preventing driver fatigue, drowsiness, and exhaust gas suicides. $\mathrm{CO}$ concentrations of $30 \mathrm{ppm}$ and oxygen levels lower than $19.5 \%$ were experienced whilst driving [22].

\subsection{Embedded and Real Time systems}

Embedded system is a field in which the terminology is inconsistent. A real time system is one in which the correctness of the computations not only depends on the accuracy of the result, but 
also on the time when the result is produced. This implies that a late is a wrong answer. A hard real time system should always respond to an event with in the deadline or else the system fails and endangers human lives but in soft real time system, failing to meet the deadline produces false output and does not endanger the human lives. All embedded systems or not real time systems and vice versa. And our designed embedded system is a soft real time system.

\subsubsection{Features of Embedded Systems}

- Multiple operations can be performed using single chip

- Fully automatic.

- Compact and Faster

\subsubsection{Components of Embedded System}

- Hardware specifically built for that application

- An embedded operating system

- User interface like push buttons, LCD, numeric displays

The major part of this project is the hardware model consisting of sufficient sensor with embedded system. Embedded systems are computer in the widest sense. Based on functionality and performance requirements, embedded systems can be categorized as, Stand-alone system, Real time system, Networked information appliances and Mobile devices. Every embedded system consists of custom-built hardware built around a central processing unit (CPU). This hardware also contains memory chips onto which the software is loaded. The software residing on the memory chip is also called as the firmware.

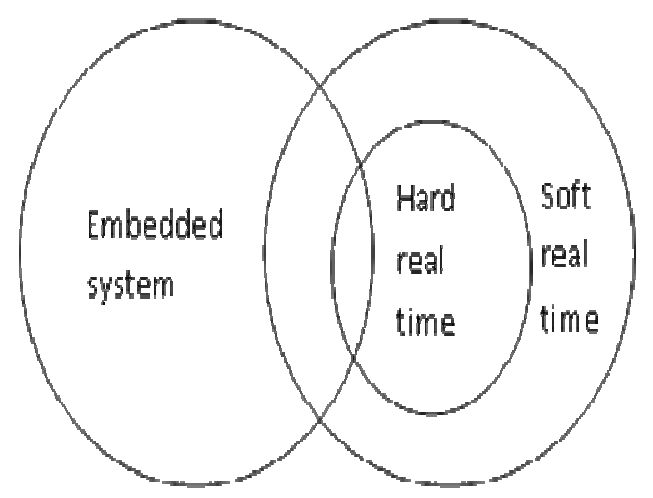

Figure: 1 Real time operating system.

\section{AIR QUALITY (AQ)}

Air quality is defined as a measure of the condition of air relative to the requirements of one or more biotic species and/or to any human need or purpose.[1] Air quality indices (AQI) are numbers used by government agencies to characterize the quality of the air at a given location. As the AQI increases, an increasingly large percentage of the population is likely to experience increasingly severe adverse health effects. To compute the AQI requires an air pollutant concentration from a monitor or model. The function used to convert from air pollutant concentration to AQI varies by pollutant, and is different in different countries. Air quality index values are divided into ranges, and each range is assigned a descriptor and a color code. Standardized public health advisories are associated with each AQI range. An agency might also encourage members of the public to take public transportation or work from home when AQI levels are high. 


\subsection{Limitations of the AQI}

Most air contaminants do not have an associated AQI. Many countries monitor ground-level ozone, particulates, sulfur dioxide, carbon monoxide and nitrogen dioxide and calculate air quality indices for these pollutants. The AQI can worsen (go up) due to lack of dilution of air emissions by fresh air. Stagnant air, often caused by an anticyclone or temperature inversion or lack of wind lets air pollution remain in a local area.

\subsection{Environmental issues in India}

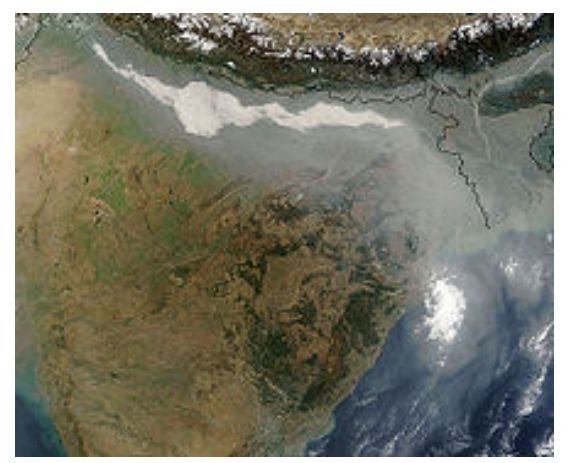

Figure: 2 Thick haze and smoke over Ganga basin

The rapid growing population and economic development is leading to a number of environmental issues in India because of the uncontrolled growth of urbanization and industrialization, expansion and massive intensification of agriculture, and the destruction of forests. Major environmental issues are forest and agricultural degradation of land, resource depletion (water, mineral, forest, sand, rocks etc.), environmental degradation, public health, loss of biodiversity, loss of resilience in ecosystems, livelihood security for the poor. Road dust due to vehicles also contributing up to $33 \%$ of an air pollution. In cities like Bangalore, around $50 \%$ of children suffer from asthma and also it causes a significant risk factor for multiple health conditions including respiratory infections, heart disease, and lung cancer, according to the WHO. One of the biggest causes of air pollution in India is from the transport system. Hundreds of millions of old diesel engines are continuously burning away diesel which has the range between 150 to 190 times the amounts of sulphur than the European diesel has.

\subsection{Necessity of $A Q$ measurement}

Air pollution levels has been monitored for over 100 years, however, it has really only been in the last 25 years that the technology has been available to measure air pollution on a real-time basis. There are several important reasons to measure air pollution are,

- Ensuring the long-term goals and targets to reduce levels of air pollution.

- Providing information to the general public about the air quality of their locality.

There are many ways of calculating the levels of air quality in an area - this can be done by estimating the rates of emissions from sources such as cars, housing estates and factories etc. However, in order to verify these calculations and get up-to-the-minute information on air 
quality, it is essential to measure air quality with sophisticated instrumentation and such a system is designed here.

\section{HARDWARE SYSTEM DESIGN}

\subsection{Block Diagram Of The Proposed System}

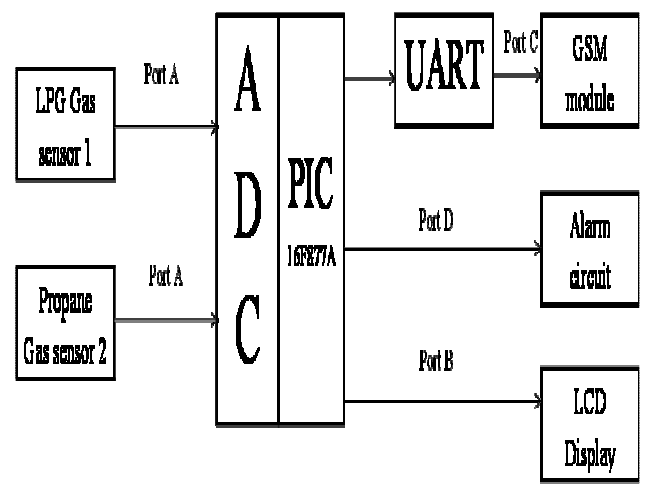

Figure: 3 Block Diagram of the Proposed System

The gas levels are sensed through the respective gas sensors (here MQ-2 and MQ-7 are used for sensing LPG and propane respectively for demonstration purpose) and sent to the PIC micro controller. The sensed analog signals are converted to digital through ADC (inbuilt in case of PIC). The sensed gas levels are displayed in the LCD; if any one gas level exceeds the set point then an alarm is generated immediately. At the same time an alert message is sent as SMS to the authorized user through the GSM modem. The block diagram of the proposed system is shown in figure3.

\subsection{GAS SENSORS}

Normally a gas sensor is the one which is made up of transducer that senses the gas molecules. It sends electrical signals as the output which is proportional to the gas concentration. The gas sensors do not sense a particular gas, thus they must tend to employ analytical techniques to adopt to identify a particular gas. However these analytical methods suffer from many disadvantages of skilled operator, specially designed PC's and slows response time etc., and the proposed system does not suffer such disadvantages. The proposed system is an automated one, but it requires to reset after every critical situation.

\subsection{LPG sensor}

It is an ideal sensor to detect the presence of a dangerous LPG leak in our home or in a service station, storage tank environment and even in vehicle which uses LPG gas as its fuel. This unit can be easily incorporated into an alarm circuit/unit, to sound an alarm or provide a visual indication of the LPG concentration. The sensor has excellent sensitivity combined with a quick response time. When the target combustible gas exist, the sensor's conductivity is higher along with the gas concentration rising.

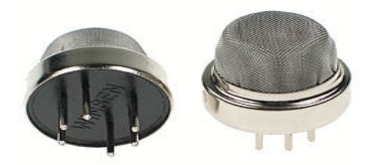

Figure: 4 LPG gas sensors 
A simple electronic circuit is used to convert change of conductivity to its corresponding output signal of gas concentration. MQ-6 gas sensor shown in figure 4 is used to sense the poisonous gas and has high sensitivity to LPG, and also response to Natural gas. It is a portable gas detector which has long life with low cost. The specification of the LPG gas sensor is shown in the table1.

Table1. Specification of LPG sensor

\begin{tabular}{|c|c|}
\hline Model No. & MQ-6 \\
\hline Sensor Type & Semiconductor \\
\hline Standard & $\begin{array}{c}\text { Bakelite } \\
\text { (Black Bakelite) }\end{array}$ \\
\hline Detection Gas & $\begin{array}{c}\text { Isobutene, Butane } \\
\text {,LPG }\end{array}$ \\
\hline Concentration & $\begin{array}{c}\text { 300-1000ppm } \\
\text { (Butane, Propane, } \\
\text { LPG) }\end{array}$ \\
\hline
\end{tabular}

\subsection{Combustible Gas sensor}

Sensitive material of MQ-2 gas sensor is $\mathrm{SnO} 2$, with lower conductivity in clean air. When the target combustible gases exist, the sensor's conductivity is higher along the gas concentration increasing. A simple electronic circuit is used to convert the change of conductivity to its corresponding output signal of gas concentration. MQ-2 gas sensor is shown in figure5, which has sensitivity to propane, butane and also to natural gas. The sensor could be used to detect different combustible gas, especially Methane and it is a low cost sensor and suitable for different applications.

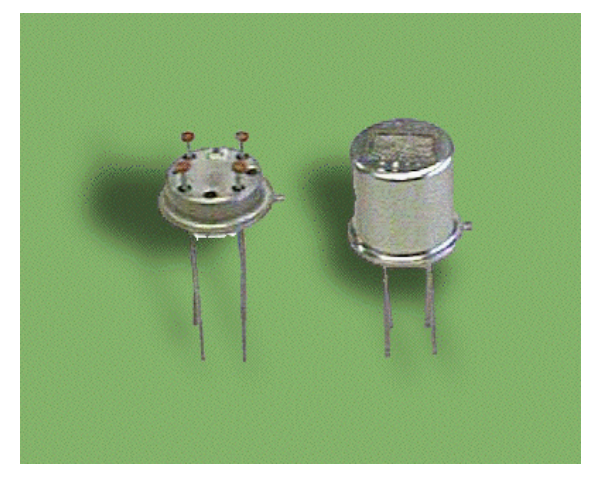

Figure: 5 Combustible gas sensors 


\subsection{GSM}

GSM stands for Global System for Mobiles. GSM provides recommendations and not requirements. The GSM module as shown in figure6 has specifications that define the functions and interface requirements in detail but not address the hardware. The reason for this is to limit the designers as little as possible but still to make it possible for the operators to buy equipment from different suppliers. The GSM network is divided into three major systems,

- The Switching system (SS)

- The Base station system (BSS)

- The Operation and support system (OSS)

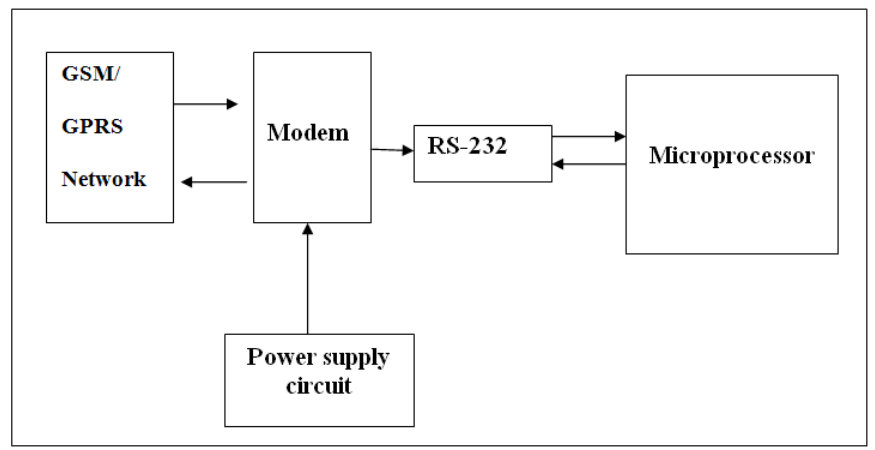

Figure: 6 Block diagram of GSM module

\subsection{PIC Microcontroller}

The Microcontroller used here is the PIC16F877. It has attractive features and they are suitable for a wide range of application. It consists of I/O ports, 3 timers, ROM, RAM, Flash memory and inbuilt ADC. PIC channel 10 bit inbuilt ADC which convert the analog value into 10 bit digital data. PIC is programmed to convert 10 bit data into an 8 bit data and to transmit the data into a transistor driver. Microcontroller 16F877A has 40 pins, 32 pins for parallel port. One port includes 8 pins, so 32 pins formed 4 parallel ports; each of them is recognized as port 0 , port 1 , port 2 and port 3. Number of each pin of parallel port starts from 0 through 7, first pin of port 0 is named P0.0 and the last pin of port 3 is named P3.7.

\subsection{PIC Features}

\subsubsection{High performance RISC CPU}

- Only 35 single word instructions to learn.

- All single cycle instructions except for program Branches which are two cycle.

- Operating speed: $20 \mathrm{MHz}$ clock input, $200 \mathrm{~ns}$ instruction cycle.

- Up to $8 \mathrm{k}$ x 14 words of FLASH program memory, up to 368 x 8 bytes of Data memory (RAM). Wide operating voltage range: $2.0 \mathrm{~V}$ to $5.5 \mathrm{~V}$

\subsubsection{Peripheral Features}

- Timer0: 8-bit timer/counter with 8-bit prescaler. 
International Journal of Distributed and Parallel Systems (IJDPS) Vol.3, No.3, May 2012

- Timer1: 16-bit timer/counter with prescaler,can be incremented during SLEEP mode.

- Timer2: 8-bit period register, prescaler and postscaler

\subsubsection{Analog Features}

- 10-bit, up to 8-channel Analog-to- Digital Convertor (A/D)

- Brownout Reset (BOR)

- Analog Comparator module with:

Two analog comparators.

$>$ Programmable on-chip voltage reference (VREF0 module.

$>$ Programmable input multiplexing from device inputs and internal voltage reference.

$>$ Comparator outputs are externally accessible.

\subsubsection{Special Micro Features}

- 100,000-erase/write cycle Enhanced Flash program memory typical.

- 1,000,000-erase/write cycle Data EEPROM memory typical.

- Data EEPROM retention $>40$ years

- Self-re-Programming under software control

- In-circuit Serial Programming (ICP) via two pins

- Watchdog Timer (WDT0 with its own on-chip RC oscillator for reliable operation.

- Programmable code protection

- Selectable saving sleep mode

- Selected oscillator options

- In-circuit Debug (ICD) via two pins 
International Journal of Distributed and Parallel Systems (IJDPS) Vol.3, No.3, May 2012

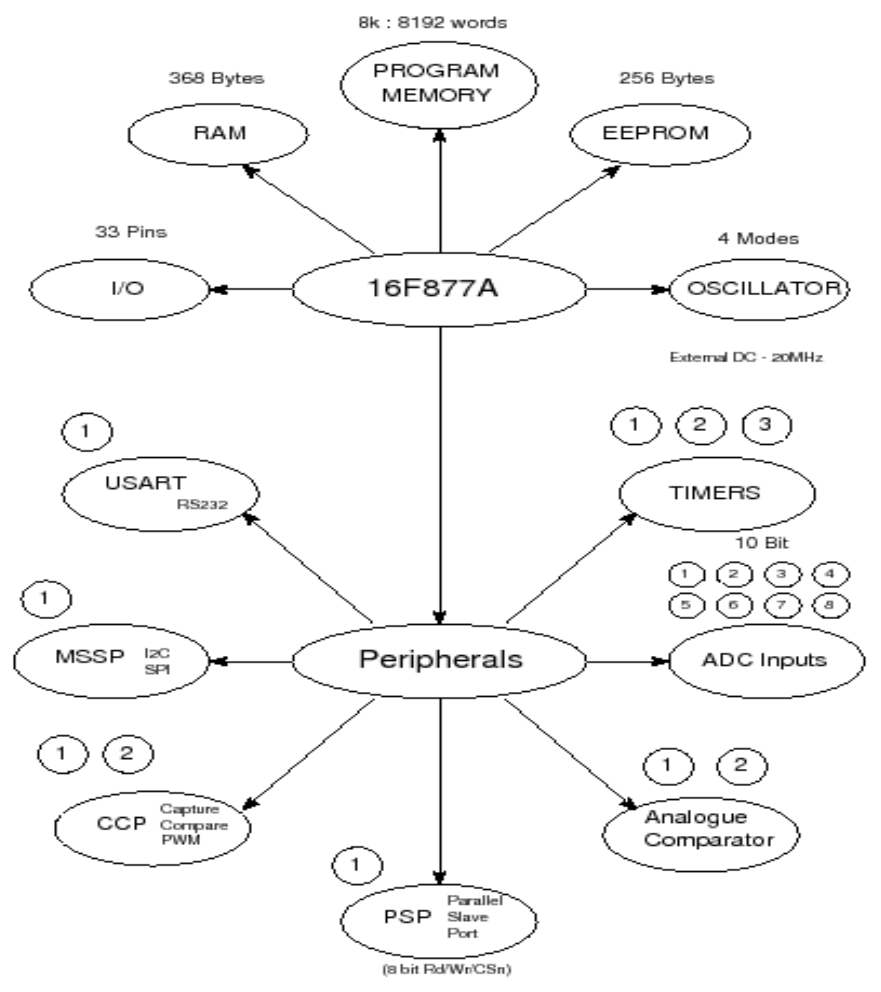

Figure: 7 PIC functional diagram

\subsubsection{Analog to digital converter(ADC)}

Analog-to-Digital conversion involves two steps: sampling and quantization. The analog signal is sampled at regular intervals and the sampled values are rounded off to fixed number of levels called quantization levels. These quantized values are converted in to bit stream. The ADC conversion of the analog input signals results in a corresponding 10-bit digital number. Many micro controllers have on-chip ADC like PIC microcontroller. If the on-chip ADC does not serve the application purpose, then an external ADC can be used. The ADC module has four registers, these four registers are:

- $\quad$ A/D Result High Register(ADRESH)

- A/D Result Low Register(ADRESL)

- $\mathrm{A} / \mathrm{D}$ Control Registe0r(ADCON0)

- A/D Control Register1(ADCON1)

The ADCON0 register controls the operations of the A/D module. The ADCON1 register configures the function of the port pins. 
International Journal of Distributed and Parallel Systems (IJDPS) Vol.3, No.3, May 2012

\section{SOFTWARE DESCRIPTION}

\subsection{Real Time Operating System}

- The RTOS (Real Time Operating System) requires only the basic functionalities of the OS which are needed for the specific application.

- The RTOS differ from other desktop OS by taking the control of application first.

- The main characteristic RTOS is its defined response time to the external stimuli.

\subsection{Software Development}

Application development on desktop computers is called native development as development and execution are done on the same hardware platform. Embedded software cannot be developed directly on the embedded system. Embedded software development is done in two stages. Initially, the software is developed on a desktop computer or a workstation. This is called the host system. Subsequently, the software is transferred to the actual embedded hardware called the target system. The host and the target system can be connected through a serial interface such as RS232 or through Ethernet. The processors of the host and the target system are generally different. Hence, this development is known as cross-platform development. The embedded software can be transferred to the target system by programming an EEPROM or Flash memory using a programmer, or downloading through a communication interface or JTAG port. There are several different ways of writing code for embedded systems depending on the complexity of the system and the amount of time and the money that can be spent. Many ready built designs provide libraries and additional software support which dramatically cut the development time. Fig 8 shows the software cross-platform development.

\begin{tabular}{|c|c|c|}
\hline HOST SYSTEM & $\begin{array}{l}\text { UNIVERSAL } \\
\text { PROGRAMMER }\end{array}$ & TARGET SYSTEM \\
\hline Debug code & $\begin{array}{c}\text { Download } \\
\text { Program }\end{array}$ & $\begin{array}{c}\text { Embedded } \\
\text { system }\end{array}$ \\
\hline Debug code & & \\
\hline Test code & & \\
\hline
\end{tabular}

\subsubsection{Programming the PIGigure: 8 Software Developments}

Step 1: Click the Start Menu and select the MPLAB IDE from the program Menu and a window will be opened.

Step 2: Click Project $\rightarrow$ new project (a window will be opened)

Step 3: Enter the PROJECT NAME, PROJECT DIRECTORY where the program to be stored in the corresponding fields and clicks ok.

Step 4: Click Configure $\rightarrow$ Select device (a window will be opened)

Step 5: Select the device name as PIC16f877 and click ok

Step 6: Click project $\rightarrow$ set language tool locations 
International Journal of Distributed and Parallel Systems (IJDPS) Vol.3, No.3, May 2012

Step 7: Expend CCS C compiler for PIC12/114/16/18 in line displayed window. Further expand the executable and select the CCS C compiler (CCSC.exe) and click ok.

Step 8: Click Project $\rightarrow$ set language suits

Step 9: Select CCS C compiler for PIC12/14/16/18 in the active tool suite and click ok

Step 10: Click file $\rightarrow$ new file. Now type the corresponding program and save it as $<$ filename.c $>$ in the corresponding location where the project name is denoted.

Step 11: Click project $\rightarrow$ add file to the project (Select the saved file and click open)

Step 12: Click project $\rightarrow$ build option $\rightarrow$ project (a window will be opened)

Step 13: Select CCS C compiler in the window, click none in the debug option, tick the use alternate settings and enter $+\mathrm{p}$ in the space provided and click ok.

Step 14: Click Project $\rightarrow$ build all the CCS compiler will denote the result if any errors indicated, go to step 10 else continue.

Step 15: Click Start menu and select the PIC ISP from the program menu and a window will be opened.

\subsubsection{Cross Compilation}

A compiler is mainly to translate program written in some human readable language into an equivalent set of opcodes for a particular processor. Each processor has its own unique machine language, and then we need to choose a compiler that is capable of producing programs for the specific target processor. In the embedded system, this compiler almost always runs on the host computer. It simply does not make sense to execute the compiler on the embedded system itself. A compiler that runs on one computer platform and produces code for another is called as cross compiler. The use of a cross compiler is one of the defining features of embedded software development, and these tools support an impressive set of host-target combinations. Toll chain for building the embedded software is shown in fig 9 . 
International Journal of Distributed and Parallel Systems (IJDPS) Vol.3, No.3, May 2012

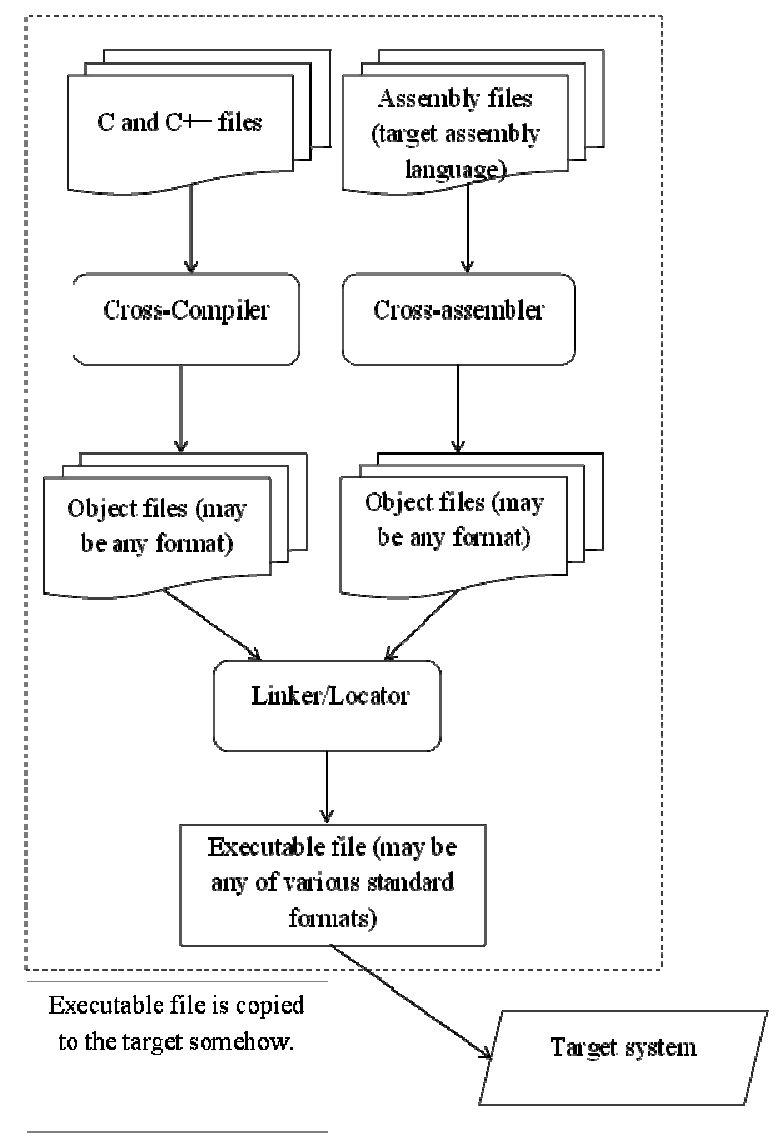

Fig.9 Tool chain for Building Embedded Software

\subsubsection{Embedded C}

One of the few constants across all these systems is the use of the $\mathrm{C}$ programming language. More than any other, $\mathrm{C}$ has become the language of embedded programmers. The $\mathrm{C}$ language has become so popular, because successful development is so frequently about selecting the best language for a given project; it is the one language that has proven itself appropriate for both 8-bit and 64-bit processors. In addition, $\mathrm{C}$ has the benefit of processor independence, which allows programmers to concentrate on algorithms and applications, rather than on the details of a particular architecture. $\mathrm{C}$ gives embedded programmers an extraordinary degree of direct hardware control without sacrificing the benefits of high-level languages. Embedded $\mathrm{C}$ is a set of language extensions for the c programming language by the $\mathrm{C}$ standards committee. It introduces a number of features not available in normal $\mathrm{C}$ and basic $\mathrm{I} / \mathrm{O}$ hardware addressing. It is having the declaration of microcontroller registers and special function as header files, we can include these files to make easy implementation. Embedded $\mathrm{C}$ has same flow and programming methodology as $\mathrm{C}$. It has unlimited number of source files, mixed $\mathrm{C}$ and assembler programming. It's Compatibility integrates into the MPLAB IDE, MPLAB ICD and most third party development tools and runs on multiple platforms: Windows, Linux, UNIX, Mac OS X, Solaris EX:- Kiel C. 


\section{IMPLIMENTATION}

The hazardous gases like LPG and combustible gas were sensed by the MQ-2 and MQ-6 sensors respectively and are monitored by the PIC microcontroller and displayed in the LCD. In critical situation, that is when the LPG exceeds from normal level above 1000ppm and in the same way when the Propane exceeds the normal level of 10000ppm then an alarm is generated and a SMS is sent to the authorized user as an alerting system, which helps in faster diffusion of the critical situation. The prototype of the proposed is shown in the Fig 10.

\section{CONCLUSION}

An embedded system for hazardous gas detection has been implemented; here only two gases (LPG and Propane) have been detected for demo purpose. The gas sensors and the critical level of the respective gas should be known, and then this system can be implemented for detecting various gases either in domestic area such as places of educational institutions, residential and industrial areas which avoids endangering of human lives. This system provides quick response rate and the diffusion of the critical situation can be made faster than the manual methods.

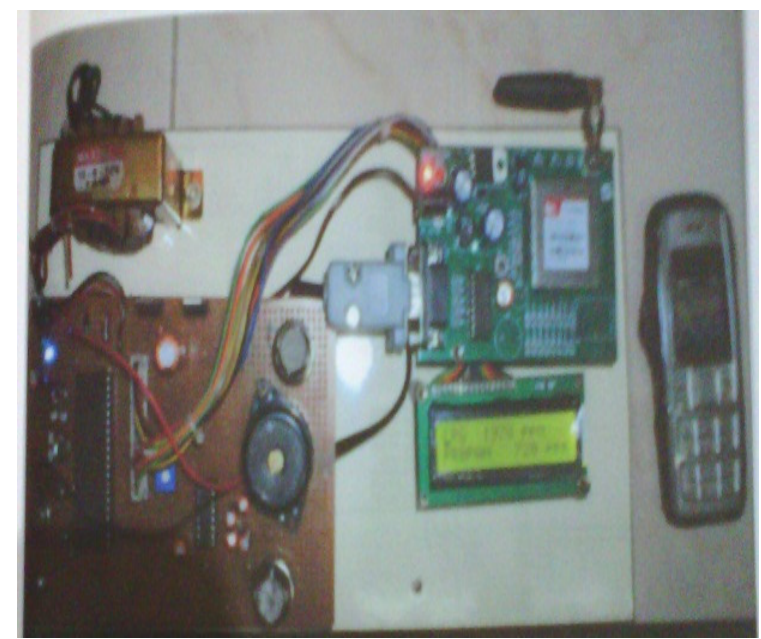

Figure: 10 Prototype of the proposed system

\section{REFERENCES}

[1]. R. Al-Ali, Member, IEEE, Imran Zualkernan, and FadiAloul, Senior Member, IEEE, “A Mobile GPRS-sensors array for Air Pollution Monitoring" vol.6, pp.410-422, Oct.2010.

[2]. NihalKularatna, Senior Member, IEEE, and B. H. Sudantha, Member, IEEE "An Environment Air Pollution Monitoring System Based on the IEEE1451 Standard for Low Cost Requirements" IEEE Sensors J., Vol. 8, pp.415-422, Apr. 2008.

[3]. M. Abu Jayyab, S. Al Ahdab, M. Taji, Z. Al Hamdani, F. Aloul, "Pollumap: Air Pollution mapper for cities", in Proc. IEEE Innovations in Information Technology Conf., Dubai, UAE, Nov.2006, pp.1-5. 
[4]. Y. J. Jung, Y. K. Lee, D. G. Lee, K. H. Ryu, and S. Nittel, "Air pollution monitoring system based on geosensor network", in Proc. IEEE Int. Geoscience Remote Sensing Symp., 2008, vol. 3, pp. 1370-1373.

[5]. M. Gao, F. Zhang, and J. Tian, "Environmental monitoring system with wireless mesh network based on Embedded System", in proc. $5^{\text {th }}$ IEEE Int. Symp. Embedded Computing, 2008, pp. 174179.

[6]. J. W. Kwon, Y. M. Park, S. J. Koo, and H. Kim, "Design of Air Pollution Monitoring system Using ZigBee Networks for ubiquitous-city", in proceedings of In. Conf. Convergence Information Technology, 2007, pp.1024-1031.

[7]. Geng Juntato, Zhou Xiaotao, Zhang Bingjie, "An Atmosphere Environment Monitor System Based on Wireless Sensor Network”, Journal of Xihua University, Natural Science, Vol. 26, no.4, pp. 44$46,2007$.

[8] F. Tsow, E. Forzani, A. Rai, R. Wang, R. Tsui, S. Mastroianni, C. Knobbe, A. J. Gandolf, and N. j. Tao, "A wearable and wireless sensor system for real-time monitoring of toxic environmental volatile organic compounds", IEEE sensors, J., vol. 9, pp. 1734-1740, Dec.2009.

[9] W. Chung and C. H. Yang, "Remote Monitoring System with Wireless Sensor Module for Room Environment", Sens. Actuators B, vol. 113, no. 1, pp. 35-42, 2009.

[10] Raj Kamal, "Embedded System Architecture Programming and Design” TATA Mc-Graw Hill.

[11] N. Kularantna and B. H. Sudantha, "An environmental air pollution monitoring system based on the IEEE 1451 standard for low cost re-quirements,"IEEE, sensors J., Vol, 8, pp. 415-422, Apr, 2008.

[12]Y. J. Jung, Y. K. Lee, D. G. Lee, k. H. Ryu, and S. Nittel, "Air Pollution monitoring system based on geo sensor network" in proc. IEEE Int. Geoscience Remote Sensing Symp., 2008, vol, 3, pp. 1370-1373. 